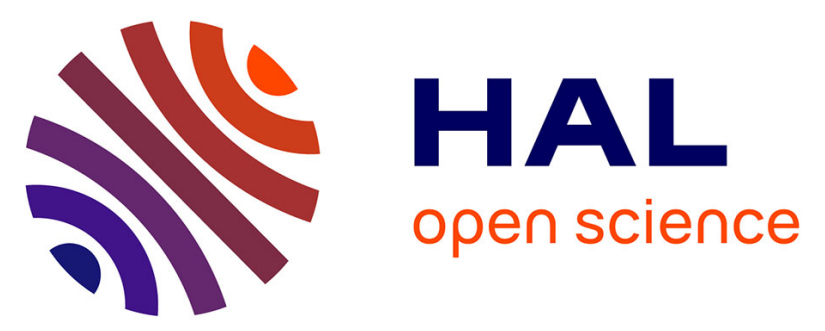

\title{
AGATHE : A tool for personalized rehabilitation of cognitive functions based on simulated activities of daily living
}

Evelyne Klinger, Abdelmajid Kadri, Eric Sorita, Jean-Luc Le Guiet, Pauline Coignard, Philippe Fuchs, Laure Leroy, Nicolas Du Lac, Fabrice Servant, Pierre-Alain Joseph

\section{To cite this version:}

Evelyne Klinger, Abdelmajid Kadri, Eric Sorita, Jean-Luc Le Guiet, Pauline Coignard, et al.. AGATHE: A tool for personalized rehabilitation of cognitive functions based on simulated activities of daily living. Innovation and Research in BioMedical engineering, 2013, 34 (2), pp.113-118. 10.1016/j.irbm.2013.01.005 . hal-01061264

\section{HAL Id: hal-01061264 https://hal.science/hal-01061264}

Submitted on 5 Sep 2014

HAL is a multi-disciplinary open access archive for the deposit and dissemination of scientific research documents, whether they are published or not. The documents may come from teaching and research institutions in France or abroad, or from public or private research centers.
L'archive ouverte pluridisciplinaire HAL, est destinée au dépôt et à la diffusion de documents scientifiques de niveau recherche, publiés ou non, émanant des établissements d'enseignement et de recherche français ou étrangers, des laboratoires publics ou privés. 


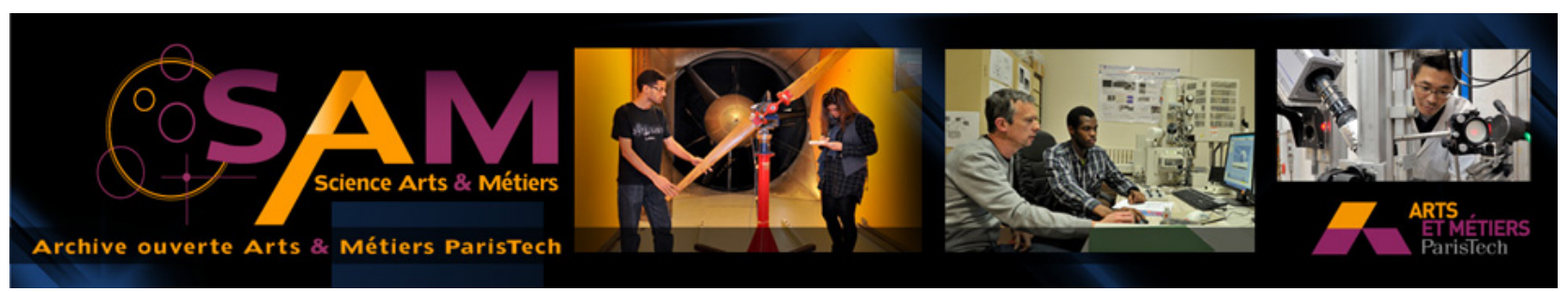

\section{Science Arts \& Métiers (SAM)}

is an open access repository that collects the work of Arts et Métiers ParisTech researchers and makes it freely available over the web where possible.

This is an author-deposited version published in: http://sam.ensam.eu

Handle ID: .http://hdl.handle.net/10985/8465

\section{To cite this version :}

Evelyne KLINGER, Abdelmajid KADRI, Eric SORITA, Jean-Luc LE GUIET, Pauline COIGNARD, Philippe FUCHS, Laure LEROY, Nicolas DU LAC, Fabrice SERVANT, Pierre-Alain JOSEPH AGATHE : A tool for personalized rehabilitation of cognitive functions based on simulated activities of daily living - IRBM - Vol. 34, n², p.113-118 - 2013 


\title{
AGATHE: A tool for personalized rehabilitation of cognitive functions based on simulated activities of daily living
}

\author{
E. Klinger ${ }^{\text {a, }}$, A. Kadri ${ }^{\text {a }}$, E. Sorita ${ }^{\text {b }}$, J.-L. Le Guiet ${ }^{\text {c }}$, P. Coignard ${ }^{\text {c }}$, P. Fuchs ${ }^{\text {d }}$, L. Leroy ${ }^{\text {d }}$, \\ N. du Lac ${ }^{\mathrm{e}}$, F. Servant ${ }^{\mathrm{f}}$, P.-A. Joseph ${ }^{\mathrm{b}}$ \\ ${ }^{a}$ LAMPA, EA 1427, arts et métiers ParisTech, 2, boulevard du Ronceray, BP 93525, 49035 Angers cedex 01, France \\ ${ }^{\mathrm{b}}$ EA 4136, CHU de Bordeaux Pellegrin, SMPR Tastet Girard, place Amélie-Raba-Léon, 33076 Bordeaux cedex, France \\ ${ }^{\mathrm{c}}$ CMRRF Kerpape, BP78, 56275 Ploemeur cedex, France \\ ${ }^{\mathrm{d}}$ Centre de robotique, Mines ParisTech, 60, boulevard Saint-Michel, 75272 Paris cedex 06, France \\ e Intempora, 2, place Jules-Gévelot, 92130 Issy-les-Moulineaux, France \\ ${ }^{\mathrm{f}}$ Dassault Systèmes, 10, rue Marcel Dassault, 78946 Vélizy-Villacoublay cedex, France
}

\begin{abstract}
Every year, tens of thousands of people fall victim to one of invalidating neurological pathologies. Acquired brain injury leads to cognitive impairment and heavy loss of autonomy. Rehabilitation interventions are needed to enable people to recover capacity and return to Instrumental Activities of Daily Living (iADL), such as grocery shopping. Unfortunately, the resources made available in cognitive rehabilitation are insufficient for the growing needs of victims of brain damage. The goal of the AGATHE project is to develop a tool that will provide therapists with an innovative means of dealing with cognitive rehabilitation and offer patients customized rehabilitation sessions, on the basis of simulated iADL. AGATHE fits into accessibility prospects of the tool (for everyone, everywhere and anywhere) and reduction of the cost of rehabilitation. AGATHE allows the strengthening and diversification of skills and expertise of the project's clinical and research partners as well as the opening of a new application field to the technology of the industrial partners. Initial tests of the AGATHE tool have been performed among therapists and patients after brain injury in order to validate usability issues. Perspective of efficacy trials has been identified and development projects are explored.
\end{abstract}

\section{Introduction}

Cognitive impairments are a major factor of loss of autonomy and dependence. In France, for a new generation of adults aged 20 to 60 years, nearly $13 \%$ of people will be affected of mental and cognitive impairment before the age of retirement from professional activity [1]. The incidence of cognitive deficits increases beyond 60 years with the impact of degenerative [2] and vascular brain disorders. Each year in France, despite the impact of road safety programs, more than 25,000 people, mostly young adults between 15 and 25 years, suffer from cognitive impairment resulting from Traumatic Brain Injury (TBI), especially after a road accident [3]. At the same time, more than 130,000 people suffer a disabling stroke [4]. Rehabilitation

\footnotetext{
* Corresponding author. Arts et Métiers ParisTech, LAMPA, 4, rue de l'Ermitage, 53000 Laval, France.

E-mail address: evelyne.klinger@ensam.eu (E. Klinger).
}

interventions are needed to enable these people to recover capacity and return to instrumental Activities of Daily Living (iADL), such as grocery shopping. Unfortunately, they are difficult to carry out and often below the expected efficacy.

Rehabilitation is a global process with sequences of assessment and rehabilitation steps [5]. Traditional rehabilitation methods lean on an analysis of the deficits and/or on a prolonged confrontation to daily living situations. If the individualized methods are the most appropriate [6,7], they are expensive to perform and time consuming. Too many patients are excluded from the access to care. It is thus necessary to find adapted and configurable tools that can be used by anyone and more often, in different places, such as rehabilitation centers, medical practices or at home. The assets of virtual reality to address this big problem of public health are today scientifically recognized [8]. So, based on immersion within virtual environments for activities of daily living, our approach takes place in the perspective of functional capacities rehabilitation and dependence reduction. 
The AGATHE project (Adaptable, configurable and upgradable tool for the generation of individualized therapeutic applications in cognitive rehabilitation, 2009-2012) ${ }^{1}$ was created in an attempt to address some of these issues. AGATHE is positioned as an industrial research project that offers a new field of application of the technologies of the industrial partners. Its finality is the integration of new habits in the therapeutic practice to diversify the propositions of intervention in cognitive impairments rehabilitation. Therapists will see their intervention facilitated by the strategies proposed by AGATHE for patient's progress, aids to success in the task, cooperation and therapeutic intensification.

\section{State of Art}

Virtual Reality (VR) offers human-computer interaction paradigm in which users are no longer simply external observers of images on a computer screen but are active participants within a computer-generated virtual world. VR provides interactive functional simulations with multimodal feedback, task graduation and review of performance thanks to various recorded data. Over the past decade, the rapid development of VR-based technologies has been both an asset and a challenge for assessment and rehabilitation [9-11]. VR allows the clinicians to immerse the patient in a spatial and temporal context difficult to provide via conventional therapy in order to create innovative clinical paradigms of rehabilitation. Moreover, VR increases patient's motivation to train thanks to gaming aspects.

In neuropsychology, studies were carried out to explore the potential of VR in various cases of cognitive impairment: attention [12], executive functions [8], memory [11], or visuospatial abilities. Some applications are also dedicated to activities of daily living in familiar places in order to train the patients before they come back home. Studies showed that virtual training was found to transfer to real task performance [13,14].

Activities of daily life, which are the core of the AGATHE project, allow the therapists to assess the patient according to various cognitive components (e.g., executive functions, memory, attention), as well as in a global functional approach. Generally, the VR-based activities are taking place in virtual kitchens [15-17], or in virtual supermarkets [18,19], the choice of the task and the quality of interaction between the subject and the virtual world being crucial. In France, the Virtual Action Planning Supermarket (VAP-S) was designed to assess executive functions and results among various populations (Parkinson Disease, stroke, or mild cognitive impairment) confirmed its feasibility and its discriminative potential [20-22]. Studies are currently carried out to test the rehabilitation assets in instrumental activities of daily living during a graduated shopping task within the VAP-S.

The observation of patient behavior and activity thanks to various recorded data (like the various patient's actions in the VR scene, but also video and audio, eye-tracking, motion capture, or physiological measurements) and the corresponding playback

\footnotetext{
1 www.agathe-rv.net.
}

capabilities, are necessary for performance review and construction of adapted interventions. In fact, the tasks in which the patient is involved allow clinicians to collect detailed information about the process of patient performance rather than focusing primarily on a final product, like a score. Consequently, the real-time acquisition and analysis of patient data are fundamental. All the possibilities of physiological and behavioral measures in a virtual environment also provide new opportunities for the investigation of human behavior and performance [23].

The use of VR for cognitive intervention is still at the stage of exploratory research with prototypes although a number of single case studies, group comparisons and even randomized clinical trials are now underway. Application of VR for healthcare is expected to continue to grow due to the unanimous and urgent need for improved assessment and rehabilitation tools [24]. The AGATHE project was created to bring an answer to this need and to provide a wide range of simulated iADL for cognitive rehabilitation.

\section{AGATHE project overview}

\subsection{Objectives}

AGATHE objective is, at first, to provide therapists with an innovative means of dealing with cognitive rehabilitation and to offer patients customized rehabilitation sessions, on the basis of simulated activities of daily living. Then it is to fit into accessibility prospects of the tool (for everyone, everywhere and anywhere) and reduction of the cost of rehabilitation. AGATHE aims at the strengthening and diversification of skills and expertise of the project's clinical and research partners as well as the opening of a new application field to the technology of the industrial partners.

\subsection{Consortium}

AGATHE project is an industrial project that was conceived and coordinated by Arts et Métiers ParisTech, due to the expertise of this project leader in virtual reality therapeutic applications. So, six partners with complementary multidisciplinary skills worked together to design and produce the AGATHE tool. The Research Team included partners from Arts et Métiers ParisTech and from Armines, both specialists in virtual reality and its challenges. The Health Team included the Bordeaux EA4136 and the Kerpape rehabilitation center, featuring specialists like rehabilitation clinicians, occupational therapists and neuropsychologists. The Industrial Team included two software publishers; Dassault Systèmes and Intempora.

\subsection{Participants}

We chose to build the AGATHE project in the context of acquired brain injury, especially in the context of Traumatic Brain Injury (TBI) and stroke. Patients and therapists who participated in the pilot tests were recruited in the rehabilitation service 


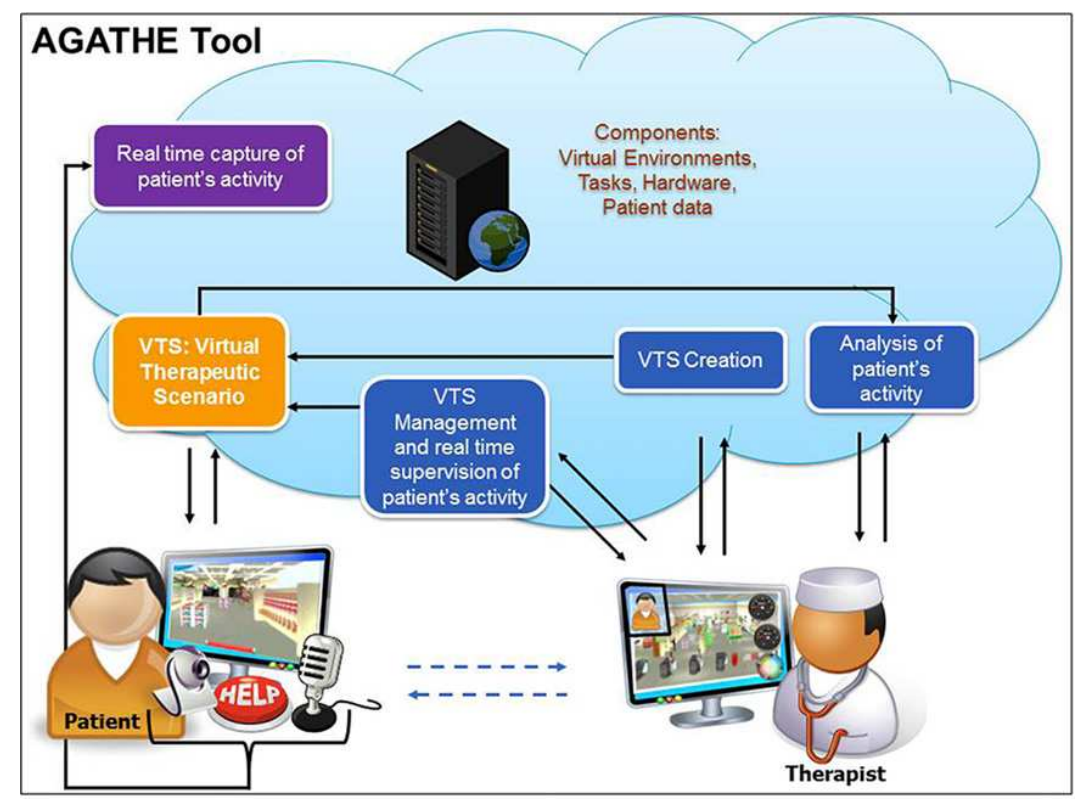

Fig. 1. AGATHE (Adaptable, configurable and upgradable tool for the generation of individualized therapeutic applications in cognitive rehabilitation) functional approach.

of the University Hospital of Bordeaux and that of Kerpape rehabilitation center.

\subsection{Method}

AGATHE tool is a software package dedicated to two types of users (patients and therapists) and based on two real-time softwares 3DVIA Studio from Dassault Systemes (creation of real-time virtual interactive environments) and RTMaps from Intempora (monitoring and real-time recording of patient activity through sensors). AGATHE tool is a Virtual Therapeutic Application (VTA) leaning on the design of Virtual Therapeutic Scenarios (VTS) that are canvas of predetermined tasks for therapeutic purposes.

The scientific and technical work was based on the arrangement of AGATHE tool in users views, corresponding to functional blocks and to the five stages through which the patient and the therapist will use the tool:

- welcome and assessment of the patient;

- design of the VTS by the therapist;

- therapeutic session within the VTS for the patient;

- supervision of the patient's activity and management of the VTS by the therapist;

- analysis of patient's activity by the therapist (Fig. 1).

This work was carried out according to the steps described below.

\subsubsection{Co-Design}

We adopted a co-design approach, which involved the whole partners. Thanks to the observation of cognitive rehabilitation sessions and to brain storming meetings, we collected and synthesized the needs and practices of therapists working in rehabilitation (physicians, neuropsychologists, occupational therapists). The designers built small VR experiences for the therapists in order to make concrete some proposals, communicate with the therapists, and collect their feedback. All this approach allowed us to draw a common thread, which served as basis for the design of AGATHE tool. For each users views, the collaborative work between care experts (researchers and therapists) and technical experts (researchers and industrial) enabled to express the functional requirements, to establish the interconnections between the five modules and to refine the usage scenarios, which ensure the validity of the tool.

\subsubsection{Research actions}

The first step of the scientific and clinical program was the definition of sequences of therapeutic activities based on data acquired by conventional programs and initial experiences of interventions supported by virtual reality. Appropriate means of facilitation and increase in difficulty of the tasks had to be established.

One of our main tasks was to provide a clear description of the chosen tasks (topographic tasks, post mail, grocery shopping) in order to model them and to implement them. Using mindmaps we described the task in a generic way according to the following components: setting, instructions and steps. We identified the critical steps in the VTS with factors of success or failure, and we extracted various therapeutic indicators from the tasks.

When a user interacts with a VR system, a lot of information is delivered to the user whose characteristics must be adapted in order to be useful to the patient. During small trials we explored some of those characteristics like the Physical Field of View (PFOV: the angle sustended by the display device and the user's retina) and the Software Field of View (SFOV: Field of View of the virtual camera), that are two fundamental parameters in VR-based experiences [25]. Some other trials allowed us to 


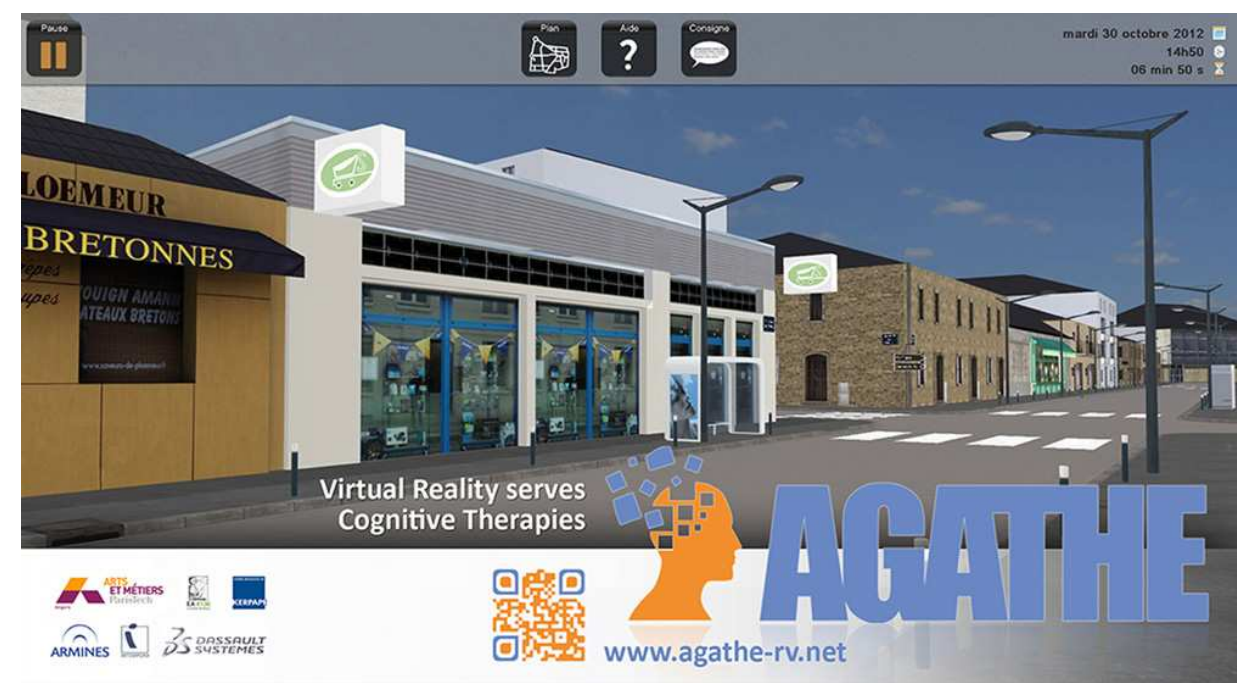

Fig. 2. AGATHE neighborhood and patient point of view.

test VR interfaces (gamepad, Kinect) and to define navigation parameters, like point of view (first or third person).

\subsubsection{Tool development}

We focused our efforts on the development of AGATHE core for first use close to the patients in care centers. This core leans on a virtual neighbourhood where functional places were positioned (town, studio, post office, supermarket) (Fig. 2). Each functional place is conducive to functional tasks or specific iADL (topographic tasks, post mail, shopping. ..). During the virtual iADL (vADL), the participant's activity is measured through indicators that can be either generic and therefore common to all tasks, such as session length, or specific to the task, as the time in a crossroads for a task topographic.

This core was built to be scalable and accept the addition of further modules: new vADLs in existing places, new places with functional tasks and specific indicators. It can record all patient's activity and replay it in real-time or delayed. It was also built to follow the patient's clinical pathway which includes various phases:

- test of abilities (to assess the capacities of the patient to success in navigating and interacting within virtual worlds);

- familiarization (to inform and train the patient about basic means of interaction in AGATHE tasks);

- evaluation (prototypical tasks to assess the status and the progress of the patient) and;

- rehabilitation interventions (customized tasks adapted to the patient's capacities and needs, and to the therapist objective).

AGATHE core relies on three software: 3DVIA Studio for the creation of the virtual entities, the implementation of the behaviors and tasks and the rendering during the patient's session, RTMaps for real-time capture of patient's activity, and Acapela for oralisation of the instructions and dialogues.

Graphical User Interfaces (GUI) have been developed around AGATHE core. Therapist GUI includes a first GUI for setting

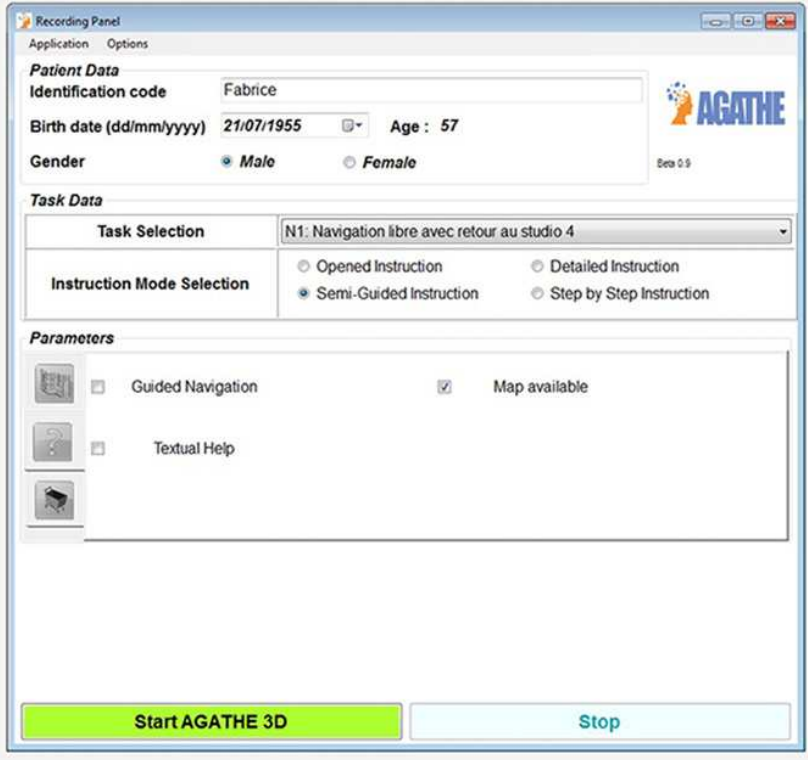

Fig. 3. Therapist Graphical User Interfaces (GUI) for setting the task.

the tasks, managing the instructions and the aids that will be delivered to the patient during the session, and a second GUI for real-time supervision of patient's activity, or delayed analysis and replay of patient's activity (Fig. 3). Patient GUI includes two main spaces: top screen is dedicated to tools potentially useful to carry out the task and linked to specific information (pause, plan of the neighbourhood, instruction, help, date and time) (Fig. 2).

All these software modules were delivered to the partners in successive beta versions that have been set up in the rehabilitation services of Bordeaux and Kerpape.

\subsubsection{Pilot trials}

During the development of the tool, preliminary tests were conducted using the navigation exercise, that is a component 


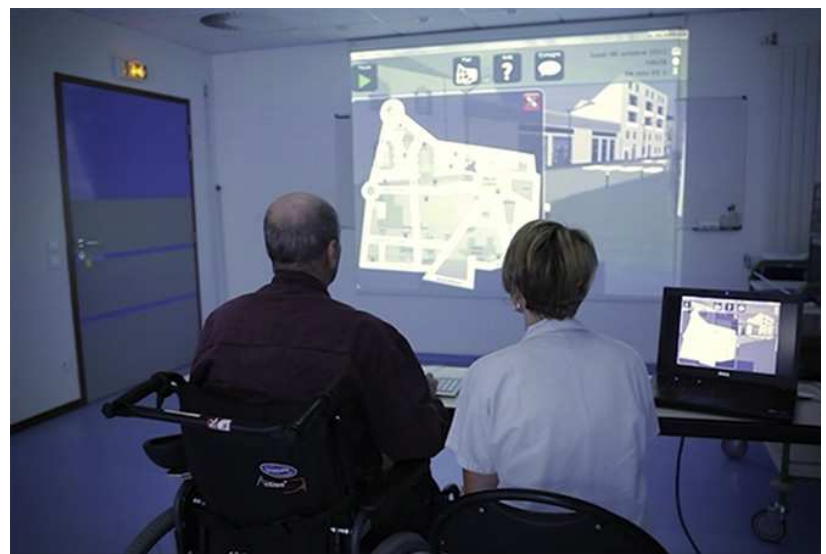

Fig. 4. Patient interacting during a therapeutic session.

of the aptitude tests. The objective was to define the optimal settings for the user point of view, the SFOV, the locomotion and rotation speeds during visual scanning, and to understand how the user estimates the distances with such parameters values. These settings are fundamental to the successful completion of tasks that are available to patients.

Two protocols (P1: therapists and P2: patients) have been established to carry out pilot tests to evaluate the feasibility and usability of the tool AGATHE with two types of end users. Protocol P1 was conducted with participants playing the role of therapist and Protocol P2 was conducted with healthy subjects playing the role of patients and then with stroke patients. During protocol P2, participants had to achieve the aptitude test, the familiarization task and two further clinical tasks, a topographical orientation task in the virtual district and an executive task, namely the mailing task. Questionnaires specific to each protocol were created to answer questions for the validation of the tool. P1-Questionnaires collect qualitative feedback about the usability of the therapist GUI (e.g. launching the software, choice and setting of the task). P2-Questionnaires collect qualitative and quantitative feedbacks about usability of patient's GUI, VR contents, or VR interfaces. An additional questionnaire allowed us to collect the feedback of the therapists about the clinical outcomes extracted from the data recorded along the session.

\section{AGATHE project results}

All the components and functionalities were tested during the pilot trials and we collected the feedback of about 15 therapists (physicians, occupational therapists, speech therapists, neuropsychologists) and 13 patients after stroke. These feedbacks contributed to the iterative process of tests and development. Therapists succeeded in customizing the experience to match patient functioning and needs, and to gradually stimulate recovery of abilities. Patients willingly invested in the attractive and rewarding rehabilitation tasks.

Finally, the first AGATHE prototype operates on a PC equipped with a good graphic card, two screens (one for the patient and one for the therapist), a webcam, and the three necessary softwares: 3DVIA Studio, RTMaps and Acapela. Navigation and interaction during the VADL are possible with various interfaces (e.g., mouse, keyboard, gamepad) (Fig. 4).

In order to guarantee on the success and on the wide distribution of AGATHE, a company is being set up to commercialize AGATHE tool. Synergies between the partners will be sustained through clinical research (efficacy trials) and development projects of new features.

\section{Conclusion}

With AGATHE, virtual reality serves cognitive therapies, by offering patients customized rehabilitation sessions, on the basis of simulated activities of daily living, and by allowing therapists to measure patient activity and to follow-up his progress. With AGATHE, patients become actors of their therapy.

AGATHE tool is now existing; preliminary data have been obtained during the pilot studies. Next steps will consist in consolidating the current prototype and increasing its functionalities, as well as assessing the efficacy of AGATHE-based rehabilitation among various populations of patients.

\section{Acknowledgement}

This work was supported by the French National Research Agency (ANR) through The TecSan program (project AGATHE ANR-09-TECS-002). The AGATHE project was certified by the Images et Réseaux cluster in 2009. We thank Laval authorities (Laval Agglomération and Conseil Général de la Mayenne) for their financial support to the Arts et Métiers ParisTech team during the AGATHE project.

\section{References}

[1] Lambert T. Déficiences et maladies invalidantes. Tendances La lettre des études de l'AGEFIPH 2007;7:1-4.

[2] Goillot C, Mormiche P. «Enquête HID en institutions de 1998. Résultats détaillés », INSEE-Résultats, série démographie et société. INSEE; 2001 $\left[n^{\circ} 83-4\right]$.

[3] Pradat-Diehl P. Dossier : les troubles cognitifs après traumatismes-crâniocérébraux sévères. Neurologies 2004;7:65-76.

[4] ANAES. Prise en charge initiale des patients adultes atteints d'accident vasculaire cérébral - Aspects paramédicaux - Argumentaire. Paris: ANAES; 2002 [118].

[5] Wilson BA. Neuropsychological rehabilitation. Annu Rev Clin Psychol 2008;4:141-62.

[6] Cicerone KD, Dahlberg C, Malec JF, Langenbahn DM, Felicetti T, Kneipp $\mathrm{S}$, et al. Evidence-based cognitive rehabilitation: updated review of the literature from 1998 through 2002. Arch Phys Med Rehabil 2005;86:1681-92.

[7] Legg L, Drummond A, Leonardi-Bee J, Gladman JR, Corr S, Donkervoort $\mathrm{M}$, et al. Occupational therapy for patients with problems in personal activities of daily living after stroke: systematic review of randomised trials. BMJ 2007;335:922.

[8] Klinger E. Virtual Reality and its contribution to the assessment and treatment of cognitive and behavioral disorders. In: Computer science. ENST: Paris; 2006 [228]

[9] Rizzo A, Kim GJ. A SWOT analysis of the field of virtual reality rehabilitation and therapy. Presence: Teleoper Virtual Environ 2005;14:119-46.

[10] Klinger E, Joseph PA. Rééducation instrumentalisée après cérébrolésion vasculaire. In: Froger J, Pélissier J, editors. Problèmes en médecine et rééducation. Paris: Masson; 2008. p. 149-65. 
[11] Rose FD, Brooks BM, Rizzo AA. Virtual reality in brain damage rehabilitation: review. Cyberpsychol Behav 2005;8:241-62 [discussion 63-71]

[12] Rizzo AA, Bowerly T, Buckwalter JG, Schultheis MT, Matheis R, Shahabi $\mathrm{C}$, et al. Virtual environments for the assessment of attention and memory processes: the virtual classroom and office. In: Sharkey P, et al, editors. Proceedings of the 4th International Conference on disability, virtual reality and associated techniques. Reading UK: University of Reading; 2002. p. 3-12.

[13] Brooks BM, McNeil JE, Rose FD, Greenwood R, Attree EA, Leadbetter A. Route learning in a case of amnesia: a preliminary investigation into the efficacy of training in a virtual environment. Neuropsychol Rehab 1999;9:63-76.

[14] Dede C. Immersive interfaces for engagement and learning. Science 2009;323:66-9.

[15] Cao X, Douguet AS, Fuchs P, Klinger E. Designing an ecological and adaptable virtual task in the context of executive functions: Preliminary study. In: Sharkey PM, Sanchez J, editors. Proceedings 8th International Conference Disability, Virtual Reality and Associated Technologies. Chile: Viña del Mar/Valparaiso; 2010.

[16] Christiansen C, Abreu B, Ottenbacher K, Huffman K, Masel B, Culpepper R. Task performance in virtual environments used for cognitive rehabilitation after traumatic brain injury. Arch Phys Med Rehabil 1998;79:888-92.

[17] Zhang L, Abreu BC, Seale GS, Masel B, Christiansen CH, Ottenbacher KJ. A virtual reality environment for evaluation of a daily living skill in brain injury rehabilitation: reliability and validity. Arch Phys Med Rehabil 2003;84:1118-24.

[18] Lo Priore C, Castelnuovo G, Liccione D. Experience with V-STORE: considerations on presence in virtual environments for effective neu- ropsychological rehabilitation of executive functions. Cyberpsychol Behav 2003;6:281-7.

[19] Rand D, Katz N, Weiss PL. Evaluation of virtual shopping in the VMall: comparison of post-stroke participants to healthy control groups. Disabil Rehabi 2007;1:1-10.

[20] Klinger E, Chemin I, Lebreton S, Marié RM. Virtual action planning in Parkinson's disease: a control study. Cyberpsychol Beha 2006;9: 342-7.

[21] Josman N, Hof E, Klinger E, Marie RM, Goldenberg K, Weiss PL, et al. Performance within a virtual supermarket and its relationship to executive functions in post-stroke patients. In: Proceedings of International Workshop on Virtual Rehabilitation; 2006 [106-9].

[22] Werner P, Rabinowitz S, Klinger E, Korczyn AD, Josman N. Use of the virtual action planning supermarket for the diagnosis of mild cognitive impairment: a preliminary study. Dement Geriatr Cogn Disord 2009;27:301-9.

[23] Astur RS, Mathalon DH, D’Souza DC, Krystal JH, Constable RT. fMRI assessment of hippocampus function in participants with schizophrenia using a Virtual Morris Water Task Cognitive Neuroscience Society Abstracts 2003.

[24] Klinger E, Weiss PL, Joseph PA. Virtual reality for learning and rehabilitation. In: Didier JP, Bigand E, editors. Rethinking physical and rehabilitation medicine. Paris: Springer; 2010. http://dx.doi.org/10.1007/9782-8178-0034-9_11

[25] Cherni H, Kadri A, Tarruella A, Joseph PA, Le Roy C, Klinger E. Virtual information display for cognitive rehabilitation: choice of screen size. J Cyber Ther Rehab 2011;4(1):73-81. 